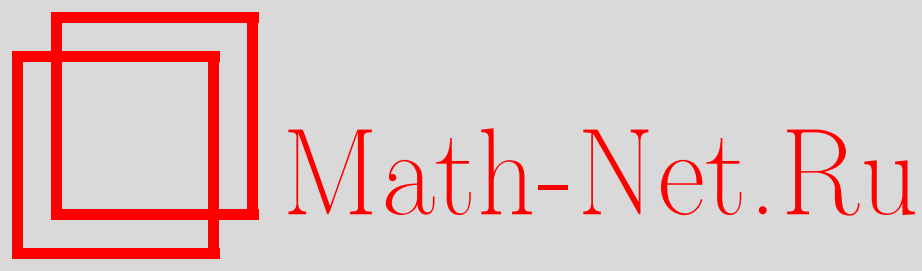

М. Д. Миссаров, А. Ф. Шамсутдинов, Зонная структура потока ренормализационной группы в фермионной иерархической модели, ТМФ, 2018, том 194, номep 3, 436-444

DOI: https://doi.org/10.4213/tmf9357

Использование Общероссийского математического портала Math-Net.Ru подразумевает, что вы прочитали и согласны с пользовательским соглашением http: //www.mathnet.ru/rus/agreement

Параметры загрузки:

IP : 3.80 .181 .102

26 апреля 2023 г., 15:43:00

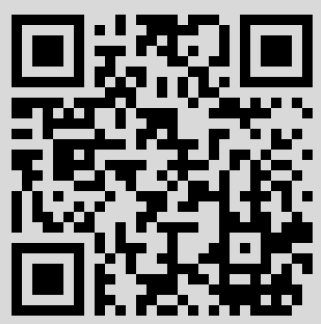




\title{
ФИЗИКА
}

Том 194, № 3

март, 2018

(C) 2018 г.

М. Д. Миссаров*, А.Ф. Шамсутдинов*

ЗОННАЯ СТРУКТУРА

\section{ПОТОКА РЕНОРМАЛИЗАЦИОННОЙ ГРУППЫ В ФЕРМИОННОЙ ИЕРАРХИЧЕСКОЙ МОДЕЛИ}

\begin{abstract}
Гауссовская часть гамильтониана четырехкомпонентной фермионной модели на иерархической решетке инвариантна относительно блок-спинового преобразования ренормализационной группы с заданной степенью нормировки (параметра ренормгруппы). Преобразование ренормгруппы в пространстве коэффициентов, задающих грассмановозначную плотность свободной меры, описывается как однородное квадратичное отображение. Это пространство трактуется как двумерное проективное пространство и визуализируется в виде диска. Если параметр ренормгруппы больше размерности решетки, то единственная притягивающая неподвижная точка ренормгруппы задается плотностью грассмановой дельта-функции. Эта неподвижная точка имеет две различные (левую и правую) инвариантные окрестности. На основании этого классифицируются точки проективной плоскости в соответствии с тем, каким образом (слева или справа) они стремятся к притягивающей точке при итерациях отображения. Обсуждается зонная структура полученных областей, и показано, что глобальный поток ренормгруппы имеет простое описание в терминах этой зонной структуры.
\end{abstract}

Ключевые слова: ренормализационная группа, фермионная модель, проективное пространство, зонная структура.

DOI: https://doi.org/10.4213/tmf9357

\section{1. ВВЕДЕНИЕ}

В работах [1]-[6] мы исследовали свойства преобразования ренормализационной группы (РГ) в рамках фермионной иерархической модели. Эта модель является фермионным (антикоммутативным) аналогом иерархической бозонной $\varphi^{4}$-модели Дайсона. Преобразование блочной РГ Вильсона в рамках бозонной иерархической модели сводится к нелинейному интегральному оператору в бесконечномерном пространстве плотностей свободной меры. Свойства этого преобразования в окрестности гауссовской неподвижной точки изучались в работах Блехера и Синая и многих

* Казанский (Приволжский) федеральный университет, Казань, Россия. E-mail: Moukadas.Missarov@kpfu.ru, aydarrrio@gmail.com 
других авторов [7]-[9]. В отличие от бозонного случая, РГ-преобразование в иерархической фермионной модели можно вычислить точно и представить в виде бирационального отображения в двухмерном пространстве констант связи модели. Это позволяет явно описать все неподвижные точки и другие динамические свойства и симметрии РГ-отображения. Отметим также, что фермионная иерархическая модель имеет естественную непрерывную $p$-адическую версию, если размер элементарного блока иерархической решетки равен простому числу $p$ (см. работы [6], [10], [11]). В этом случае такие проблемы квантовой теории поля, как ультрафиолетовые расходимости и процедура перенормировки имеют естественную интерпретацию в терминах классической теории динамических систем.

В статье [4] мы описали некоторые свойства глобальной динамики потока РГ в плоскости констант связи модели $(r, g)$. Анализируя поток РГ с помощью компьютера, мы обнаружили, что почти все точки верхней полуплоскости $\{(r, g): g>0\}$, являющейся инвариантной относительно РГ-отображения, при последовательных итерациях этого отображения уходят в бесконечность "слева" $(r \rightarrow-\infty, g \rightarrow \infty)$ или "справа" $(r \rightarrow \infty, g \rightarrow \infty)$. Было обнаружено, что множества точек, уходящих "налево" или "направо", имеют сложную фрактальную структуру, и эти результаты привели к описанию сложного критического поведения в этой модели. Для получения глобальной картины динамики РГ мы использовали представление модели в проективном пространстве. Недавно мы исследовали РГ-преобразование и разработали простой алгоритм анализа глобальной динамики непосредственно в проективном пространстве [12], [13]. В данной работе мы представляем результаты компьютерных экспериментов, полученные с помощью этого алгоритма. Мы классифицируем точки проективной плоскости в соответствии с тем, каким образом итерации РГ-отображения этих точек стремятся к единственной притягивающей неподвижной точке. Мы явным образом описываем зонную структуру множеств, полученных в результате такой классификации. Компьютерные эксперименты показывают, что глобальная динамика РГ имеет красивое описание в терминах этой структуры. В частности, проясняется глобальное поведение устойчивых инвариантных кривых для различных неподвижных точек. Заметим, что РГ в евклидовых моделях исследуется в низших порядках теории возмущений в окрестности гауссовской неподвижной точки. Иерархическая фермионная модель интересна тем, что она предоставляет уникальную возможность описать глобальный РГ-поток во всей плоскости констант связи. Явное описание свойств РГ в рамках иерархической фермионной модели порождает ряд нетривиальных гипотез для иерархических и евклидовых бозонных моделей [14].

Пусть $p$ является натуральным числом, $p>1$. Иерархическую решетку $T_{p}^{d}$ можно определить как решетку $Z^{d}$ целочисленных $d$-мерных векторов, снабженную иерархическим расстоянием $d_{p}(i, j)$. Пусть $k=\left(k_{1}, \ldots, k_{d}\right) \in Z^{d}, s \in \mathbb{N}$, $V_{k, s}=\left\{j \in Z^{d}:\left(k_{l}-1\right) p^{s}<j_{l} \leqslant k_{l} p^{s}, l=1, \ldots, d\right\}$. Иерархическое расстояние $d_{p}(i, j), i, j \in Z^{d}$, определяется как $d_{p}(i, j)=p^{s(i, j)}$, если $i \neq j$,

$$
s(i, j)=\min \left\{s: \text { существует } k \text { такое, что } i \in V_{k, s}, j \in V_{k, s}\right\} .
$$

В случае, когда $p$ - простое число, решетку $T_{p}^{d}$ можно представить как решетку чисто дробных $d$-мерных $p$-адических векторов с $p$-адическим расстоянием между ними [6]. Фермионное поле определяется набором четырехкомпонентных спинов 
$\psi^{*}(i)=\left(\bar{\psi}_{1}(i), \psi_{1}(i), \bar{\psi}_{2}(i), \psi_{2}(i)\right), i \in T_{p}^{d}$, компоненты которых являются образующими алгебры Грассмана, а модель задается гамильтонианом

$$
H\left(\psi^{*} ; \alpha\right)=H_{0}\left(\psi^{*} ; \alpha\right)+\sum_{i \in T_{p}^{d}} L\left(\psi^{*} ; r, g\right) .
$$

Здесь гауссовская часть гамильтониана определяется как

$$
\begin{gathered}
H_{0}\left(\psi^{*} ; \alpha\right)=\sum_{i, j \in T_{p}^{d}} d_{0}(i, j)\left[\bar{\psi}_{1}(i) \psi_{1}(j)+\bar{\psi}_{2}(i) \psi_{2}(j)\right], \\
d_{0}(i, j)=\frac{1-p^{\alpha-d}}{1-p^{-\alpha}} d_{p}^{-\alpha}(i, j), \quad i \neq j, \quad d_{0}(i, i)=\frac{1-p^{-d}}{1-p^{-\alpha}} .
\end{gathered}
$$

Негауссовская часть гамильтониана определяется лагранжианом

$$
L\left(\psi^{*}(i) ; r, g\right)=r\left(\bar{\psi}_{1}(i) \psi_{1}(i)+\bar{\psi}_{2}(i) \psi_{2}(i)\right)+g \bar{\psi}_{1}(i) \psi_{1}(i) \bar{\psi}_{2}(i) \psi_{2}(i),
$$

где $r$ и $g$ - вещественнозначные константы связи, а $\alpha$ - вещественнозначный параметр модели. В случае, когда $\alpha=2+d$, гамильтониан $H_{0}\left(\psi^{*} ; 2+d\right)$ является иерархической версией гауссовского гамильтониана, задаваемого оператором Лапласа. В физической литературе в евклидовых моделях обычно рассматриваются гамильтонианы именно такого вида, а корреляционные функции негауссовских полей анализируются в терминах формальных разложений по параметру $\epsilon=4-d$, где $d$ - размерность пространства. Но для полноты и математической ясности картины естественно считать параметры $\alpha$ и $d$ свободными. Блок-спиновое преобразование РГ Вильсона определяется как

$$
r(\alpha) \psi^{*}(i)=p^{-\alpha / 2} \sum_{j \in V_{i, 1}} \psi^{*}(j),
$$

где $\alpha$ - параметр РГ. РГ-преобразование не изменяет структуру гамильтониана и сводится к преобразованию $R(\alpha)$ в плоскости констант связи $R(\alpha)(r, g)=\left(r^{\prime}, g^{\prime}\right)$ :

$$
r^{\prime}=\lambda\left(\frac{(r+1)^{2}-g}{(r+1)^{2}-g / n}(r+1)-1\right), \quad g^{\prime}=\frac{\lambda^{2}}{n}\left(\frac{(r+1)^{2}-g}{(r+1)^{2}-g / n}\right)^{2} g,
$$

где $\lambda=p^{\alpha-d}, n=p^{d}-$ количество вершин в элементарной ячейке иерархической решетки $T_{p}^{d}$.

В дальнейшем мы описываем преобразование РГ в пространстве коэффициентов разложения грассмановозначной “плотности” свободной меры

$$
f\left(\psi^{*}\right)=e^{-L\left(\psi^{*} ; r, g\right)} .
$$

В общем случае эта плотность задается в виде

$$
f\left(\psi^{*} ; c\right)=c_{0}+c_{1}\left(\bar{\psi}_{1} \psi_{1}+\bar{\psi}_{2} \psi_{2}\right)+c_{2} \bar{\psi}_{1} \psi_{1} \bar{\psi}_{2} \psi_{2},
$$

$c=\left(c_{0}, c_{1}, c_{2}\right) \in \mathbb{R}^{3}$. В регулярном случае, когда $c_{0} \neq 0$, константы связи $r$ и $g$ связаны с коэффициентами $c$ соотношениями

$$
r(c)=-\frac{c_{1}}{c_{0}}, \quad g(c)=\frac{c_{1}^{2}-c_{0} c_{2}}{c_{0}^{2}} .
$$


Если $c_{0}=0$ (как, например, в случае, когда плотность является грассмановой $\delta$-функцией $\left.\delta\left(\psi^{*}\right)=\bar{\psi}_{1} \psi_{1} \bar{\psi}_{2} \psi_{2}\right)$, экспоненциальное представление для плотности невозможно.

Тройка $\left(c_{0}, c_{1}, c_{2}\right)$ естественным образом трактуется как точка двумерного вещественного проективного пространства $R P^{2}$, поскольку две тройки, отличающиеся общим ненулевым множителем, задают одно и то же гиббсовское состояние.

Обозначим РГ-преобразование в пространстве коэффициентов $c$ так же, как $R(\alpha)$ :

$$
\begin{gathered}
R(\alpha)\left(c_{0}, c_{1}, c_{2}\right)=\left(c_{0}^{\prime}, c_{1}^{\prime}, c_{2}^{\prime}\right), \quad c_{0}^{\prime}=\left(\left(c_{1}-c_{0}\right)^{2}+\frac{1}{n}\left(c_{0} c_{2}-c_{1}^{2}\right)\right), \\
c_{1}^{\prime}=\lambda\left(\left(c_{1}-c_{0}\right)\left(c_{2}-c_{1}\right)+\frac{1}{n}\left(c_{0} c_{2}-c_{1}^{2}\right)\right), \quad c_{2}^{\prime}=\lambda^{2}\left(\left(c_{2}-c_{1}\right)^{2}+\frac{1}{n}\left(c_{0} c_{2}-c_{1}^{2}\right)\right) .
\end{gathered}
$$

$\mathrm{B}(r, g)$-координатах преобразование $R(\alpha)$ имеет тривиальную (гауссовскую) неподвижную точку $(0,0)$ и две негауссовские неподвижные точки $\left(r_{+}(\alpha), g_{+}(\alpha)\right.$ ) ("плюсовую" неподвижную точку) и $\left(r_{-}(\alpha), g_{-}(\alpha)\right)$ ("минусовую" неподвижную точку) при условии $\alpha \neq d$ :

$$
\begin{aligned}
r_{+}(\alpha) & =\frac{p^{d / 2}-p^{\alpha-d}}{1-p^{d / 2}}, & g_{+}(\alpha) & =\frac{r_{+}(\alpha)\left(1+r_{+}(\alpha)\right)^{2}}{1+r_{+}(\alpha)+p^{-d / 2}}, \\
r_{-}(\alpha) & =\frac{-p^{d / 2}-p^{\alpha-d}}{1+p^{d / 2}}, & g_{-}(\alpha) & =\frac{r_{-}(\alpha)\left(1+r_{-}(\alpha)\right)^{2}}{1+r_{-}(\alpha)-p^{-d / 2}} .
\end{aligned}
$$

Заметим, что "плюсовая" неподвижная точка является фермионным аналогом евклидовой негауссовской неподвижной точки, которая строится в рамках формального $(4-d)$-разложения. В $c$-координатах гауссовская неподвижная точка задается вектором $(1,0,0)$. В $c$-координатах можно видеть еще одну неподвижную точку $(0,0,1)$, которая соответствует плотности свободной меры, задаваемой грассмановой $\delta$-функцией $\delta\left(\psi^{*}\right)=\bar{\psi}_{1} \psi_{1} \bar{\psi}_{2} \psi_{2}$. Обозначим эту неподвижную точку $\delta$. Других неподвижных точек отображение $R(\alpha)$ при $\alpha \neq d$ не имеет. В дальнейшем мы предполагаем, что $\alpha>d$, и можно показать, что при этом условии точка $\delta$ является единственной притягивающей неподвижной точкой. Отображение $R(\alpha)$ из $R P^{2}$ в $R P^{2}$ определено всюду, за исключением точки $(1,1,1)$, которую мы называем сингулярной.

\section{2. ЗОННАЯ СТРУКТУРА ПОТОКА РГ}

Рассмотрим реализацию проективного $c$-пространства в виде полусферы

$$
S=\left\{\left(c_{0}, c_{1}, c_{2}\right): c_{0}^{2}+c_{1}^{2}+c_{2}^{2}=1, c_{0} \geqslant 0\right\},
$$

в которой противоположные точки граничной окружности $c_{1}^{2}+c_{2}^{2}=1$ отождествлены. Для получения плоской (двумерной) картины мы используем ортогональную проекцию $S$ на диск $D=\left\{\left(c_{1}, c_{2}\right): c_{1}^{2}+c_{2}^{2} \leqslant 1\right\}$. Регулярная точка $(r, g)$ соответствует точке $\left(c_{1}(r, g), c_{2}(r, g)\right)$,

$$
c_{1}(r, g)=-\frac{r}{\sqrt{1+r^{2}+\left(r^{2}-g\right)^{2}}}, \quad c_{2}(r, g)=\frac{r^{2}-g}{\sqrt{1+r^{2}+\left(r^{2}-g\right)^{2}}},
$$




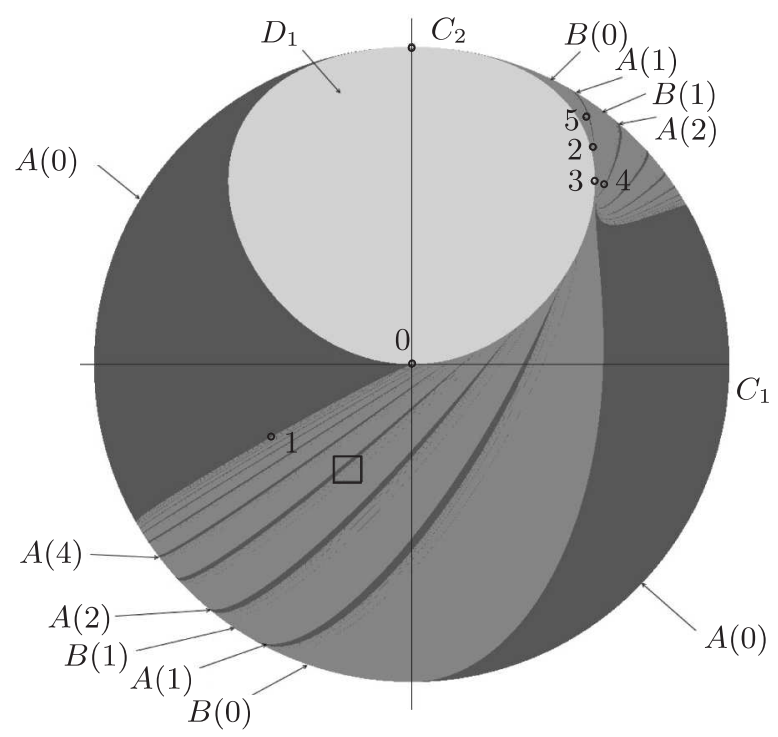

Рис. 1. Диск $D$ и РГ-инвариантные области притяжения неподвижной точки $\delta=(0,1)$ при $\alpha=1,7, p=2, d=1$.

принадлежащей внутренней части диска $D$. Тривиальная неподвижная точка $(r, g)=$ $(0,0)$ также представлена на диске $D$ точкой $(0,0)$. Неподвижная точка $\delta$ в $\left(c_{1}, c_{2}\right)$ координатах задана точкой $(0,1)$. РГ-инвариантная линия $g=0$ в $\left(c_{1}, c_{2}\right)$-координатах соответствует кривой $l_{0}=\left\{\left(c_{1}(r, 0), c_{2}(r, 0)\right) ; r \in R\right\}$. Пополняя кривую $l_{0}$ предельной точкой $(0,1)$, мы получаем замкнутую кривую $l$. Нижняя полуплоскость $\{(r, g): g<0\}$ задана на диске $D$ областью $D_{1}$, ограниченной кривой $l$ (см. рис. 1 ). Верхняя полуплоскость $\{(r, g): g>0\}$ отображается на внутреннюю часть области $D \backslash D_{1}$. Обозначим

$$
\begin{gathered}
V_{\mathrm{L}}=\left\{\left(c_{1}, c_{2}\right) \in D \backslash D_{1}: c_{1} \leqslant 0, c_{2} \geqslant \frac{4}{\sqrt{21}}\right\}, \\
V_{\mathrm{R}}=\left\{\left(c_{1}, c_{2}\right) \in D \backslash D_{1}: c_{1} \geqslant 0, c_{2} \geqslant \frac{3+b_{0}}{\sqrt{1+\left(3+b_{0}\right)^{2}}}\right\}, \quad b_{0}=-\frac{\lambda n-\lambda}{\lambda n-1} .
\end{gathered}
$$

Области $V_{\mathrm{L}}$ и $V_{\mathrm{R}}$ имеют общую точку $(0,1)$, которая соответствует неподвижной точке $\delta$. В работе [13] мы доказали, что обе эти области инвариантны относительно РГ-отображения и лежат в области притяжения неподвижной точки $\delta$. На основании этого был предложен алгоритм (подробнее см. статью [13]), который позволяет классифицировать почти все точки области $D \backslash D_{1}$ в соответствии с тем, каким образом они стремятся при итерациях отображения РГ к неподвижной точке $\delta$. Если за конечное количество итераций РГ точка $\left(c_{1}, c_{2}\right)$ попадает в область $V_{\mathrm{L}}$, то мы окрашиваем ее в темно-серый цвет и говорим, что она стремится к неподвижной точке $\delta$ слева. Если же за конечное количество итераций РГ точка $\left(c_{1}, c_{2}\right)$ попадает в область $V_{\mathrm{R}}$, то мы окрашиваем ее в серый цвет и говорим, что она стремится к неподвижной точке $\delta$ справа. На рис. 1 изображен диск $D$ и РГ-инвариантные области притяжения неподвижной точки $\delta=(0,1)$ в случае, когда $\alpha=1,7, p=2$, 


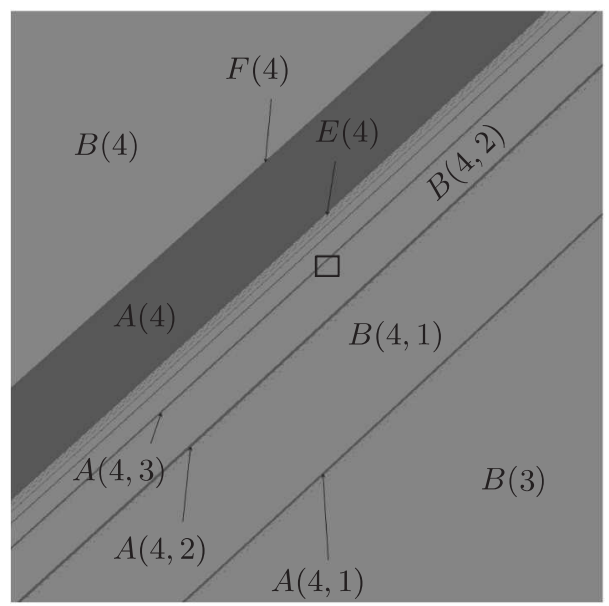

Рис. 2. Увеличенное изображение квадрата, выделенного на рис. 1.

$d=1$. Гауссовская неподвижная точка обозначена как точка 0, "плюсовая" и "минусовая" неподвижные точки обозначены точками 1 и 2, сингулярная точка - точкой 3. Нижняя полуплоскость $\{(r, g): g<0\}$ в $c$-координатах отображается на область $D_{1}$, которая окрашена в светло-серый цвет. Мы не обсуждаем в настоящей работе РГ-динамику в этой области. Соответственно верхняя полуплоскость $\{(r, g): g>0\}$ отображается на область $D \backslash D_{1}$. Почти все точки области $D \backslash D_{1}$ при итерациях РГ-отображения стремятся к неподвижной точке $\delta$. Точки, окрашенные в темно-серый цвет, при итерациях РГ-отображения стремятся к точке $\delta$ слева (оставаясь в области $D \backslash D_{1}$ ), точки, окрашенные в серый цвет, стремятся к $\delta$ справа. В темно-серой зоне мы видим большую область $A(0)$ и последовательность более мелких непересекающихся областей $A(1), A(2), A(3), \ldots$ Эти области расположены друг за другом, и чем больше номер области, тем ближе эта область расположена к основной области $A(0)$.

Будем называть области $A(i), i=1,2, \ldots$, сателлитными областями области $A(0)$. В свою очередь каждая из областей $A(i)$ имеет свою собственную счетную серию сателлитных непересекающихся областей $A(i, j), j=1,2, \ldots$ Далее, области $A(i, j)$ имеют свои собственные серии сателлитных зон $A(i, j, k), k=1,2, \ldots$, и т. д. (см. рис. 2 и рис. 3). На первый взгляд область $A(0)$ выглядит как объединение двух различных связных областей, но на самом деле $A(0)$ является связной областью, потому что граничные сегменты этих областей, лежащие на окружности $c_{1}^{2}+c_{2}^{2}=1$, расположены в точности друг против друга и, следовательно, представляют одно и то же подмножество точек в проективной плоскости. То же самое верно и для всех сателлитных областей. В серой зоне мы видим ту же самую картину основную область $B(0)$ и счетную серию непересекающихся сателлитных областей $B(1), B(2), B(3), \ldots$, их сателлитные серии $B(i, j), i=1,2, \ldots, j=1,2, \ldots$, и т. д. Из рис. 2,3 видно, что области $A\left(i_{1}, i_{2}, \ldots, i_{k}\right)$ и $B\left(i_{1}, i_{2}, \ldots, i_{k}\right)$ имеют общую граничную линию, которую мы обозначим $F\left(i_{1}, i_{2}, \ldots, i_{k}\right)$. Этот тип граничной линии можно охарактеризовать как “четкую" границу, потому что любой достаточно малый диск, центрированный в любой точке такой граничной линии, делится 


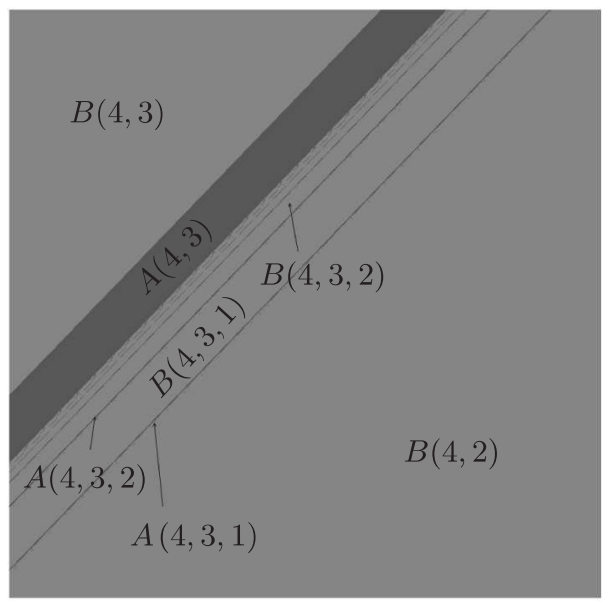

Рис. 3. Увеличенное изображение квадрата, выделенного на рис. 2.

этой линией на серую и темно-серую области одинакового размера. Локально области $A\left(i_{1}, i_{2}, \ldots, i_{k}\right)$ и $B\left(i_{1}, i_{2}, \ldots, i_{k}\right)$ выглядят как полосы, и мы видим, что эти полосы также имеют граничные линии "размытого" типа. Это означает, что любой достаточно малый диск, центрированный в любой точке "размытой" граничной линии, делится этой линией на одноцветную область и двухцветную область. Мы обозначаем "размытую" граничную линию области $A\left(i_{1}, i_{2}, \ldots, i_{k}\right)$ как $E\left(i_{1}, i_{2}, \ldots, i_{k}\right)$, a "размытую" граничную линию области $B\left(i_{1}, i_{2}, \ldots, i_{k}\right)-$ как $G\left(i_{1}, i_{2}, \ldots, i_{k}\right)$.

Рис. 2 является увеличенным изображением квадрата, выделенного на рис. 1 . На рис. 2 мы видим области $A(4), B(4), B(3)$ и их сателлитные зоны $A(4,1), A(4,2)$, $B(4,1), B(4,2)$ и т. д. Мы видим также "четкую" граничную линию $F(4)$ между $A(4)$ и $B(4)$ и "размытую" граничную линию $E(4)$. Рис. 3 является увеличенным изображением квадрата, выделенного на рис. 2. Здесь мы видим области $A(4,3), B(4,2)$ и их сателлиты $A(4,3,1), A(4,3,2), B(4,3,1), B(4,3,2)$ и т. д. Компьютерные эксперименты показывают, что если точка принадлежит области $A\left(i_{1}, i_{2}, \ldots, i_{k}\right)$ и $i_{1}>1$, то после одной РГ-итерации она переходит в область $A\left(i_{1}-1, i_{2}, \ldots, i_{k}\right)$. Если $i_{1}=1$, то точка переходит в область $A\left(i_{2}, i_{3}, \ldots, i_{k}\right)$. Точка из области $A(i), i=1,2, \ldots$, переходит в область $A(i-1)$ за одну РГ-итерацию. Схематически мы можем описать динамику РГ в темно-серой зоне следующей диаграммой:

$$
\begin{aligned}
A\left(i_{1}, i_{2}, \ldots, i_{k}\right) & \rightarrow A\left(i_{1}-1, i_{2}, \ldots, i_{k}\right) \rightarrow \cdots \rightarrow A\left(1, i_{2}, \ldots, i_{k}\right) \rightarrow \\
& \rightarrow A\left(i_{2}, \ldots, i_{k}\right) \rightarrow \cdots \rightarrow A\left(i_{3}, i_{4}, \ldots, i_{k}\right) \rightarrow \cdots \rightarrow A(1) \rightarrow A(0) .
\end{aligned}
$$

Диаграмма РГ-динамики в серой зоне имеет такой же вид:

$$
\begin{aligned}
B\left(i_{1}, i_{2}, \ldots, i_{k}\right) & \rightarrow B\left(i_{1}-1, i_{2}, \ldots, i_{k}\right) \rightarrow \cdots \rightarrow B\left(1, i_{2}, \ldots, i_{k}\right) \rightarrow \\
& \rightarrow B\left(i_{2}, \ldots, i_{k}\right) \rightarrow \cdots \rightarrow B\left(i_{3}, i_{4}, \ldots, i_{k}\right) \rightarrow \cdots \rightarrow B(1) \rightarrow B(0) .
\end{aligned}
$$

Наконец, РГ-динамика на граничных линиях $F\left(i_{1}, i_{2}, \ldots, i_{k}\right), E\left(i_{1}, i_{2}, \ldots, i_{k}\right)$, $G\left(i_{1}, i_{2}, \ldots, i_{k}\right)$ имеет аналогичное описание. Компьютерные эксперименты показывают, что линии $F\left(i_{1}, i_{2}, \ldots, i_{k}\right)$ являются частями устойчивой РГ-инвариантной кривой для неподвижной точки $\delta$. Линии $G\left(i_{1}, i_{2}, \ldots, i_{k}\right)$ являются частями 
устойчивой РГ-инвариантной кривой для "минусовой" неподвижной точки, линии $E\left(i_{1}, i_{2}, \ldots, i_{k}\right)$ являются частями устойчивой РГ-инвариантной кривой для “плюсовой" неподвижной точки при $\alpha>3 d / 2$ и устойчивой РГ-инвариантной кривой для гауссовской неподвижной точки при $3 d / 2 \geqslant \alpha>d$. Эксперименты показывают, что почти все точки области $D \backslash D_{1}$ окрашиваются в серый или темно-серый цвет. Но существует также дискретное (конечное или бесконечное) множество неокрашенных точек. Например, можно описать явным образом нетривиальный цикл РГ-отображения второго порядка. Пусть

$$
\begin{gathered}
\left.t_{ \pm}(\alpha)=\left(\lambda^{-1 / 2}+\lambda^{1 / 2}\right)^{-2}\left[\left(\left(\lambda^{-1}+\lambda n^{-1}\right)\left(\lambda+\lambda^{-1} n^{-1}\right)\right)^{1 / 2} \mp\left(1+n^{-1}\right)\right)\right] \\
s(\alpha)=\left(\frac{\lambda+n^{-1} \lambda^{-1}}{\lambda^{-1}+\lambda n^{-1}}\right)^{1 / 2},
\end{gathered}
$$

где $\lambda=p^{\alpha-d}, n=p^{d}$. Пусть

$$
\begin{aligned}
r_{4}(\alpha) & =\frac{1+t_{+}(\alpha)}{s(\alpha) t_{+}(\alpha)-1}, & r_{5}(\alpha) & =\frac{1-t_{-}(\alpha)}{s(\alpha) t_{-}(\alpha)-1}, \\
g_{4}(\alpha) & =\frac{t_{+}(\alpha)\left(s(\alpha)^{1 / 2}+s(\alpha)^{-1 / 2}\right)^{2}}{\left(t_{+}(\alpha) s(\alpha)-1\right)^{2}}, & g_{5}(\alpha) & =\frac{t_{-}(\alpha)\left(s(\alpha)^{1 / 2}-s(\alpha)^{-1 / 2}\right)^{2}}{\left(t_{-}(\alpha) s(\alpha)-1\right)^{2}} .
\end{aligned}
$$

Тогда точки $\left(r_{4}(\alpha), g_{4}(\alpha)\right)$ и $\left(r_{5}(\alpha), g_{5}(\alpha)\right)$ образуют нетривиальный цикл второго порядка для отображения $R(\alpha)$ в $(r, g)$-пространстве. На рис. 1 эти точки в $\left(c_{1}, c_{2}\right)$ пространстве обозначены точками 4 и 5 для случая $\alpha=1,7, p=2, d=1$. Компьютерные эксперименты показывают, что точки 4 и 5 не принадлежат множествам типа $A, B, E, F, G$.

Многочисленные эксперименты показывают, что такая же структура зон и поведение глобального потока РГ остаются справедливыми и для других значений $\alpha>d$, $p>1$ и $d>1$.

\section{Список литературы}

[1] E. Yu. Lerner, M. D. Missarov, "Fixed points of renormalization group for the hierarchical fermionic model", J. Statist. Phys., 76:3-4 (1994), 805-817.

[2] М.Д. Миссаров, "РГ-инвариантные кривые в фермионной иерархической модели", TMФ, 114:3 (1998), 323-336.

[3] М. Д. Миссаров, "Критические явления в фермионной иерархической модели", ТМФ, 117:3 (1998), 471-488.

[4] M. D. Missarov, "Dynamical phenomena in the hierarchical fermionic model", Phys. Lett. A, 253:1-2 (1999), 41-46.

[5] M. D. Missarov, "Renormalization group solution of fermionic Dyson model", Asymptotic Combinatorics with Application to Mathematical Physics (NATO Advanced Study Institute, St. Petersburg, Russia, July 9-22, 2001), NATO Science Series II: Mathematics, Physics and Chemistry, 77, eds. V. A. Malyshev, A. M. Vershik, Kluwer, Dordrecht, 2002, 151-166.

[6] М. Д. Миссаров, "Непрерывный предел в фермионной иерархической модели", ТМФ, 1999, № 1, 40-50.

[7] P. M. Bleher, Ja. G. Sinai, "Investigation of the critical point in models of the type of Dyson's hierarchical models", Commun. Math. Phys., 33:1 (1973), 23-42.

[8] Я. Г. Синай, Теория фазовых переходов, Наука, М., 1980. 
[9] P. Collet, J.-P. Eckmann, A Renormalization Group Analysis of the Hierarchical Model in Statistical Mechanics, v. 74, Lecture Notes in Physics, Springer, Berlin, 1978.

[10] В. С. Владимиров, И. В. Волович, Е. И. Зеленов, $p$-Адический анализ и математическая физика, Физматлит, М., 1994.

[11] B. Dragovich, A. Yu. Khrennikov, S. V. Kozyrev, I. V. Volovich, "On p-adic mathematical physics", p-Adic Numbers Ultrametric Anal. Appl., 1:1 (2009), 1-17.

[12] М.Д. Миссаров, "Ренормализационная группа в фермионной иерархической модели в проективных координатах", ТМФ, 173:3 (2012), 355-362.

[13] М.Д. Миссаров, А.Ф. Шамсутдинов, “Алгоритм исследования динамики ренормализационной группы в проективном пространстве", Тр. МИАН, 285 (2014), 221-231.

[14] M. D. Missarov, " $p$-Adic renormalization group solutions and the Euclidean renormalization group conjectures", p-Adic Numbers Ultrametric Anal. Appl., 4:2 (2012), 109-114.

Поступила в редакцию 27.02.2017 